\title{
Physical Activity and Cardiovascular Disease: Directions for Future Research
}

\author{
Mark Hamer* and Emmanual Stamatakis
}

Department of Epidemiology and Public Health, University College London, UK

\begin{abstract}
Regular physical activity is widely accepted as playing a crucial role in cardiovascular disease (CVD) prevention. However, despite extensive research in this field there are crucial gaps in our knowledge. This paper discusses future research directions including population subgroup-specific exercise doses, research in under represented populations, the role of cardiorespiratory fitness in predicting CVD risk, and novel biological mechanisms that might mediate the inverse association between physical activity and CVD risk.
\end{abstract}

Regular physical activity is widely accepted as playing a crucial role in cardiovascular disease (CVD) prevention [16]. Current physical activity recommendations for the general population advocate the accumulation of at least 30 minutes of moderate intensity physical activity on five or more days a week or vigorous activity at least three times per week [7]. However, despite extensive research in this field there are crucial gaps in our knowledge. Several important areas have emerged that include examining population subgroup-specific exercise doses that are required for primary and secondary CVD prevention; the association between physical activity and CVD in under-represented populations such as women, children, ethnic minorities, and clinical patient groups; the role of physical activity and cardiorespiratory fitness (CRF) in predicting future risk for CVD, and novel biological and physiological mechanisms that might mediate the inverse association between physical activity and CVD risk.

There is surprisingly little epidemiological evidence on the cardio-protective effects of physical activity in relation to current recommendations. In a large prospective study of over 200,000 participants, those meeting the physical activity recommendations had the lowest risk of CVD, although those that met the recommendations through vigorous activity benefited to a greater degree (32\% risk reduction) than those meeting recommendations through moderate activity (27\% risk reduction) [8]. One of the key messages of these recommendations is that activities performed during daily living, (e.g. walking or cycling, stair-climbing, gardening, home improvement work, intense housework) may be sufficient for the prevention of CVD and premature death. Although the evidence on the health benefits of walking, cycling, or stair-climbing is compelling, the degree of protection conferred from intense domestic physical activity is less clear and future research must address this issue. Several large scale prospective cohort studies in men suggest that vigorous activity is more beneficial for protection against CVD [3,4]. For example, in the Health Professional's follow up study, men who ran for an hour or more per week had a

\footnotetext{
*Address correspondence to this author at the Department of Epidemiology and Public Health, University College London, 1-19 Torrington Place, London WC1E 6BT, UK; Tel: (44) 207679 5969, Fax: (44) 207916 8542, E-mail:m.hamer@ucl.ac.uk
}

$42 \%$ CVD risk reduction compared with $18 \%$ lowered risk in men that only walked briskly for half an hour per day [4]. These associations, however, have not been substantiated in women. In the Nurse's Health Study, for example, both moderate and vigorous intensity exercise provided similar levels of protection against CVD [2]. It is possible that the disparate results reflect differences in training adaptations between men and women or given that levels of vigorous exercise in women was relatively low compared with men, the findings may reflect a lack of range of vigorous exercise in women to detect significant effects. Interestingly, exercise intensity has not been associated with any of the favourable training effects on key risk factors such as HDL cholesterol and blood pressure $[9,10]$, thus it is possible that a threshold effect may exist for some risk factors or that there is an intensity versus volume trade-off.

The role of regular exercise and the dose of activity required to achieve benefit in specific populations is not well established. In participants with existing CVD, for example, light or moderate activity was more beneficial than vigorous sporting activities for protection against CVD mortality [11]. Nevertheless, recent training studies in cardiac patients suggest that high intensity or high volume exercise training is more beneficial in reversing left ventricular remodeling, diminishing expression of atherogenic adhesion molecules, and improving aerobic capacity, endothelial function, and quality of life in comparison with a moderate exercise intensity/volume interventions $[12,13]$. Emerging evidence is beginning to uncover an association between physical activity and CVD risk factors in children [14], which may have significance in adulthood [15]. Therefore, future research in under represented populations is critical.

The precise mechanisms through which physical activity lowers CVD risk are incompletely understood. Even after adjustments for traditional CVD risk factors, such as blood pressure, lipids, and adiposity, the inverse association between physical activity and CVD persists. Recent research has examined the effects of physical activity on novel risk factors, such as inflammatory and hemostatic markers and glucose metabolism. There is presently little information, however, about the relative contribution of various risk factors to activity related reduction in CVD risk. In the only study to date that has examined this issue, known risk factors explained a large proportion (59\%) of the inverse association 
between physical activity and incident CVD events in women, with inflammatory and haemostatic risk factors and blood pressure making the largest contribution [16]. Thus, future studies of this nature will be required to confirm and extend these findings.

Low CRF and low physical activity levels are independently associated with an increased risk for CVD and CHD. Although the exact mechanisms through which physical activity and fitness interact and jointly relate to CVD risk are not clear [17], there are studies showing that CRF [18-21] and physical activity [22] predict CVD risk equally well or better than conventional CVD risk factors such as blood pressure, high density and total cholesterol, smoking, and body mass index. Unlike these conventional risk factors, however, CRF and physical activity are not routinely assessed in primary care settings to identify individuals at high risk for CVD. This is mainly due to practical considerations (e.g. lack of resources to perform exercise testing in primary care), the widespread belief held by the medical establishment that physical activity modification is not possible [23], and the lack of randomised controlled trial evidence showing that improvement in physical activity or CRF will lead to proportional decreases in CVD risk. Given that existing methods for estimating future CVD risk and taking preventive action often perform poorly [24] these are promising areas of research that need to be expanded. For example, an international group of experts have proposed a method to assess CRF in healthcare settings using non-exercise testing variables that are relatively easy and inexpensive to obtain, which include gender, age, body mass index, resting pulse rate and self-reported physical activity [25].

In summary, future research is required in critical areas to further extend our knowledge about the cardio-protective effects of physical activity. Future advances in the measurement of physical activity will also provide researchers with more accurate estimates of exposure on a population level that may help to resolve unanswered issues.

\section{ACKNOWLEDGEMENTS}

The authors receive grant funding from the British Heart Foundation, UK (MH) and the National Institute for Health Research, UK (ES).

\section{REFERENCES}

[1] Davey Smith G, Shipley MJ, Batty GD, et al. Physical activity and cause-specific mortality in the Whitehall study. Public Health 2000; 114: 308-315.

[2] Manson JE, Hu FB, Rich-Edwards JW, et al. A prospective study of walking as compared with vigorous exercise in the prevention of coronary heart disease in women. N Engl J Med 1999; 341: 650658.

[3] Lee IM, Sesso HD, Oguma Y, et al. Relative intensity of physical activity and risk of coronary heart disease. Circulation 2003; 107: 1110-1116.

[4] Tanasescu M, Leitzmann MF, Rimm EB, et al. Exercise type and intensity in relation to coronary heart disease in men. JAMA 2002; 288: 1994-2000.
[5] Leon AS, Myers MJ, Connett J. Leisure time physical activity and the 16-year risks of mortality from coronary heart disease and allcauses in the Multiple Risk Factor Intervention Trial (MRFIT). Int J Sports Med 1997; 18: S208-S215.

[6] Hamer M, Chida Y. Walking and primary prevention. A metaanalysis of prospective cohort studies. Br J Sports Med (E-pub ahead of print) doi:10.1136/bjsm.2007.039974

[7] Haskell WL, Lee IM, Pate RR, et al. Physical activity and public health: updated recommendation for adults from the American College of Sports Medicine and the American Heart Association. Circulation 2007; 116: 1081-1093.

[8] Leitzmann MF, Park Y, Blair A, et al. Physical activity recommendations and decreased risk of mortality. Arch Intern Med 2007; 167: 2453-2460.

[9] Cornelissen VA, Fagard RH. Effects of endurance training on blood pressure, blood pressure-regulating mechanisms, and cardiovascular risk factors. Hypertension 2005; 46: 667-675.

[10] Kodama S, Tanaka S, Saito K, et al. Effect of aerobic exercise training on serum levels of high-density lipoprotein cholesterol: a meta-analysis. Arch Intern Med 2007; 167: 999-1008.

[11] Wannamethee SG, Shaper AG, Walker M. Physical activity and mortality in older men with diagnosed coronary heart disease. Circulation 2000; 102: 1358-1363.

[12] Wisløff U, Støylen A, Loennechen JP, et al. Superior cardiovascular effect of aerobic interval training versus moderate continuous training in heart failure patients: a randomized study. Circulation 2007; 115: 3086-3094.

[13] Peschel T, Sixt S, Beitz F, et al. High, but not moderate frequency and duration of exercise training induces downregulation of the expression of inflammatory and atherogenic adhesion molecules. Eur J Cardiovasc Prev Rehabil 2007; 14: 476-482.

[14] Andersen LB, Harro M, Sardinha LB, et al. Physical activity and clustered cardiovascular risk in children: a cross-sectional study (The European Youth Heart Study). Lancet 2006; 368: 299-304.

[15] Raitakari OT, Juonala M, Kähönen M, et al. Cardiovascular risk factors in childhood and carotid artery intima-media thickness in adulthood: the Cardiovascular Risk in Young Finns Study. JAMA 2003; 290: 2277-2283.

[16] Mora S, Cook N, Buring JE, et al. Physical activity and reduced risk of cardiovascular events: potential mediating mechanisms. Circulation 2007; 116: 2110-2118.

[17] Blair SN, Cheng Y, Holder JS. Is physical activity or physical fitness more important in defining health benefits? Med Sci Sports Exerc 2001; 33: S379-S399.

[18] Laukkanen JA, Kurl S, Salonen R, et al. The predictive value of cardiorespiratory fitness for cardiovascular events in men with various risk profiles: a prospective population-based cohort study. Eur Heart J 2004; 25: 1428-1437.

[19] Myers J, Prakash M, Froelicher V, et al. Exercise capacity and mortality among men referred for exercise testing. New Eng J Med 2002; 346: 793-801.

[20] Aktas MK, Ozduran V, Pothier CE, et al. Global risk scores and exercise testing for predicting all-cause mortality in a preventive medicine program. JAMA 2004; 292: 1462-1468.

[21] Mora S, Redberg RF, Sharrett AR, et al. Enhanced risk assessment in asymptomatic individuals with exercise testing and Framingham risk scores. Circulation 2005; 112: 1566-1572.

[22] Hu G, Tuomilehto J, Borodulin K, et al. The joint associations of occupational, commuting, and leisure-time physical activity, and the Framingham risk score on the 10-year risk of coronary heart disease. Eur Heart J 2007; 28: 492-498.

[23] Ornish D. Statins and the soul of medicine. Am J Cardiol 2002; 89: 1286-1290.

[24] Brindle P, Beswick A, Fahey T, et al. Accuracy and impact of risk assessment in the primary prevention of cardiovascular disease: a systematic review. Heart 2006; 92: 1752-1759.

[25] Jurca R, Jackson AS, LaMonte MJ, et al. Assessing cardiorespiratory fitness without performing exercise testing. Am J Prev Med 2005; $29: 185-193$. 